\title{
Effectiveness of Multimodal Intervention on Audiological and Psycho-Social Profile of Meniere's Disease: A Case Study
}

\section{Pragnya P Prusty ${ }^{1}$, Santosh Kumar Swain ${ }^{2}$, Satyabrata Panigrahi ${ }^{3 *}$, Sibananda Mishra ${ }^{4}$ and Ramkrishna Pattanayak ${ }^{5}$}

${ }^{1}$ Clinical Audio-Vestibular Specialist, Department of Audio-vestibular Sciences, Institute of Health Sciences, Bhubaneswar, Odisha, India

${ }^{2}$ Professor and Head, Department of ENT, IMS and Sum Hospital, Siksha 'O'

Anusandhan University, Bhubaneswar, Odisha, India

${ }^{3}$ Lecturer, Department of Audiology and Speech-Language Pathology, Institute of Health Sciences, Bhubaneswar, Odisha, India

${ }^{4}$ Professor, Department of Psychology, Institute of Health Sciences, Bhubaneswar, Odisha, India

${ }^{5}$ Department of Yogic Rehabilitation, Institute of Health Sciences, Bhubaneswar, Odisha, India

*Corresponding Author: Satyabrata Panigrahi, Lecturer, Department of Audiology and Speech-Language Pathology, Institute of Health Sciences, Bhubaneswar, Odisha, India.
Received: August 24, 2021

Published: September 06, 2021

(C) All rights are reserved by Satyabrata

Panigrahi., et al.

\section{Abstract}

Meniere's disease (MD) is a chronic disorder of the inner ear that is characterized by intermittent attacks of loud tinnitus, a sense of pressure in the ear, vertigo and progressive permanent hearing loss in one or both ears. Anxiety and dizziness occur as co-morbid symptoms in a larger percentage of patients than would be expected from chance alone. The individual profile of Meniere's disease in different patients makes it difficult to diagnose on the basis of symptomatology alone. The impact of Meniere's disease on quality of life has highlighted the importance of an additional approach to support the diagnosis and intervention of Meniere's disease. In this study, we discussed about the interface between Meniere's disease, its audiological manifestations and psycho-social factors influencing the Quality of life. The two conditions are linked by overlapping neural circuits that include monoaminergic pathways and the parabrachial nucleus network, and they are functionally related via both somatopsychic and psychosomatic mechanisms. A unique strategy for patient management is discussed in this paper. The results confirmed that vertigo can the most debilitating symptom in Meniere's disease. Correlating the clinical features of subject with Audiologic, Vestibular, Yogic and Psychological evaluation highlighted the clinical value of an interdisciplinary team approach for diagnosis and intervention.

Effective patient management requires a better understanding of the physiological and psychological nature of the disease, as well the relationship that exists between the factors. The development of a generally acceptable plan for diagnosis and treatment poses a challenge for clinicians involved in the care of this condition. Therefore, we must constantly update our knowledge through interdisciplinary teamwork and research for the benefit of our patients with the help of increased understanding of the pathophysiology and psychophysiology of MD patient, management may become more effective.

Keywords: Meniere’s Disease; Multimodal Intervention; Hearing Rehabilitation; Pharmacological Treatment; Yogic Rehabilitation 


\section{Abbreviations}

MD: Meniere's Disease; VHI: Vertigo Handicap Inventory; DHI: Dizziness Handicap Inventory; QoL: Quality of Life; SCAT: Sinha's Comprehensive Anxiety Test; PTA: Pure Tone Audiometry; SD: Speech Discrimination; OAEs: Oto-acoustic Emissions; VNG: Videonystagmography; EcoG: Electrocochleography; VSS: Vertigo Symptom Scale; VOR: Vestibulo-ocular Reflex; vHIT: Video Head Impulse Test; PMR: Progressive muscle Relaxation

\section{Introduction}

Meniere's disease is a disorder of the inner ear characterized by recurrent attacks of vertigo, fluctuating sensorineural hearing loss, and tinnitus [1] with an incidence of 15 - 50 people per population of 100,000 people [2]. These individuals often exhibit high levels of anxiety on initial presentation compared with patients with hearing loss, tinnitus, otalgia, or dizziness due to other causes. Associated traits may include a fear of spontaneous vertiginous attacks, preoccupation with the details of symptoms, and sensitivity to stress-related events making patients unable to participate in activities of daily life and to interact with their social environment, such as work and education. Although vertigo spells frequently prevent patients from performing planned daily activities and may be more disabling in some patients depending on vocational and coping factors, the threat of vertigo attacks may also affect activities and create a sense of despair [3].

Clinical studies indicate that a stressful lifestyle, as well as the stress-modulating hormone vasopressin, are associated with inner ear fluid homeostasis disorder and subsequent inner ear hydrops [1]. Another factor is that Meniere's patients' mental health issues may make it difficult for them to communicate effectively with their doctors [4]. Mental health care is considered to play an important role in good therapeutic results.

In our study, the diagnosis of MD is based on the History, Clinical picture, medical investigations, Psychological and Audio-vestibular examinations. An audiological test battery included the following tests: Pure Tone Audiometry (PTA), Speech discrimination, Otoacoustic emissions (OAEs), Videonystagmography (VNG), Electrocochleography (EcochG), General, Symptom specific (Vertigo Handicap Inventory) and Self-reported (Dizziness Handicap Inventory) questionnaires.

The psychosomatic investigations involved an interview and the psychological state was evaluated clinically by the use of ques- tionnaires to evaluate quality of life (QoL). As personality measure, we used (i) The Mental Status Examination developed by David R. Norris, Molly S. Clark, Sonya Shipley which measures patient's psychological functioning at a given point in time, under the domains of appearance, attitude, behavior, mood, and affect, speech, thought process, thought content, perception, cognition, insight, and judgment and (ii) Manual for Sinha's Comprehensive Anxiety Test- SCAT.

Pharmacological treatment, Dietary intervention, Audiological, Psychological and Yogic Rehabilitation were scheduled and planned accordingly.

\section{Clinical history}

Patient complained of Ringing sensation of perceived high frequency in Right ear for 2 years without any vertigo episodes. Episodes of vertigo attacks started 6 months ago. No fluctuations in tinnitus perceived in pitch and intensity even after any vertigo episodes. The tinnitus is constant and is not bothersome during the day, but is disturbing during the night as reported. Slight heaviness and Reduced hearing sensitivity in the Right ear since last 8 months. His vertigo was defined as active as the patient had experienced at least one episode of severe vertigo within 3 months of the psychological battery as he suffered persistent disequilibrium.

Non-auditory impact

Frequency of vertigo: Before medications: Daily lasting $5 \mathrm{sec}-$ onds; During medications: No vertigo attacks as reported; After medication discontinued: Every second day lasting 3 - 5 seconds.

\section{Commencement, nature, duration of the attack:}

- $\quad$ First episode: $2^{\text {nd }}$ May, 2020. He was at his workplace (manages a shop). He was unsteady, Rotational vertigo, nausea was reported. No jerky movement/Head thrust.

- Worst episode: $10^{\text {th }}$ October, 2020. Blackout, vomiting, heaviness in the head, Rotational vertigo, sudden jerk of head and movement on the left side.

- $\quad$ Last episode: $6^{\text {th }}$ December, 2020. Blackout, Rotational vertigo, 5 secs.

Frequency and number of attacks: Since last 6 months, he is having vertigo attacks, sometimes daily \& sometimes in every alternate day. No. of attacks per day: 1 - 2 . 
Auditory impact:

- Hearing loss in right ear.

- Tinnitus: High frequency tinnitus.

- Neurological symptoms: No history of headache, loss of consciousness, diplopia, dysarthria, facial numbness. Fall-Once.

- Autonomous symptoms: Heart palpitations increases during the episode. BP remains normal as reported.

- $\quad$ Aggravating/mitigating factors including medications: During medications, vertigo attack reduced drastically. Post discontinuation, vertigo relapsed.

- $\quad$ Current medications: No.

\section{Emotional impact}

Apart from the incapacitating effects of the physical manifestations, He is emotionally disturbed. He feels a sense of loss of control over his life and this leads to a certain degree of uncertainty and instability in his psychological well-being. Interference in sleep pattern (evaluated through sleep calendar), change in social contact and performance at work has led to significant anxiety and depression.

\section{Problem statement and rationale}

Review of literature on Meniere's Disease revealed that the diversified and individual course that MD takes in different patients makes it difficult for medical clinicians to identify the disease on the basis of symptomatology alone. It may take some time before the diagnosis is confirmed and too many patients live with chronic symptoms without knowing the diagnosis. The mean interval between the age at onset and the age at definite diagnosis is three years. The disease itself is unpleasant, but it may be even more stressful for the patient to have no explanation for the symptom. This increases the patient's stress level, lowering his or her quality of life even further.

The impact of MD on quality of life has highlighted the significance of quantitative physiological tests as an additional tool to support MD diagnosis. There is to date no single diagnostic protocol on which a definite diagnosis of MD can be based, and therefore it is important to establish the diagnostic value of different Multimodal tests that enable clinicians to compile effective test batteries. Insight into multi-disciplinary approach will allow clinicians to arrive at the critical diagnosis more rapidly, resulting in more effective treatment regimen.
Study design

Experimental Single Case Study using Audio-vestibular, QoL, Psychological, and social life instruments administered over 18 months in patient undergoing multi-modal treatment for Meniere's disease.

\section{Materials and Methods}

A 32-year-old male adult patient with Meniere's disease visited the Department of Audio-vestibular Sciences, Institute of Health Sciences, Odisha, India. The study protocol was approved by local institutional review boards. Patient gave informed consent. The study was conducted in compliance with the protocol, Good Clinical Practices, and applicable regulatory requirements. The diagnosis of Meniere's Disease was established by:

1. Comprehensive clinical history.

2. Physical examination: Otoscopy, blood pressure, pulse rate, orthostatic hypotension, etc.

3. Diagnostic testing which included a battery of audio-vestibular and psychological tests.

Audiological evaluation: Recent areas of interest in diagnosis of MD have focused on auditory and vestibular test results and relating them to the pathophysiology of the disease:

- Pure tone audiometry (PTA) (air- and bone conduction), Speech discrimination (SD) scores, Oto-acoustic emissions (OAEs) and Videonystagmography (VNG). Specific tests for detection of endolymphatic hydrops included Electrocochleography (EcoG) and dehydrating agents such as Glycerol in combination with audiological tests. Dehydrating drugs were used to lower endolymphatic pressure in the inner ear and cause a detectable effect, such as an increase in pure tone thresholds (PTTs).

- $\quad$ PTA: The air conduction PT audiometry was performed using the MAICO Audiometer GmbH MA52 and MAICO TDH-39 headphones. The above-mentioned audiometer was used in conjunction with the Radioear B-71 bone conduction receiver to perform bone conduction PT audiometry. Hearing function was assessed using a pure tone audiometer and the four-tone average $(\mathrm{a}+\mathrm{b}+\mathrm{c}+\mathrm{d}) / 4(\mathrm{a}, \mathrm{b}, \mathrm{c}$, and $\mathrm{d}$ are hearing levels at $0.25,0.5,1$, and $2 \mathrm{kHz}$, respectively) according to the modified AAOHNS criteria of 1995. 
- $\quad$ OAE analyzer: The MAICO ERO•SCAN was used to perform DPOAE and TEOAE testing.

- VNG: Videonystagmography was undertaken using the device (Balance Eye ${ }^{\circledR}$ ). Balance Eye is a binocular VNG system which is class 2A European CE certified. It has the provision for eye movement recording with vision allowed and denied. Oculomotor activities like saccades, smooth pursuit, gaze with and without fixation and responses to horizontal high frequency headshake, hyperventilation, lateral canal head impulse test, and positional tests were used to evaluate vestibular function.

- EcochG: The BIO-LOGIC Evoked Potential program system (Version 5.00 Model) was used to evoke electrocochleographic responses. Omni-Prep surgical scrub and gauze pads were used to prepare the subject's skin before the electrodes were applied. Ten20 conductive EEG paste was used on the two surface electrodes. A tympanic membrane electrode with FCG electrode gel was used to record EcoG results.

- Dizziness handicap inventory: (DHI; Jacobson and Newman, 1990 ) is one of the most widely used self-reported measures for evaluating vestibular disorders. This measure provides an understanding of the self-perceived disability imposed by vestibular system disease. DHI is a 25-item scale with 3-point rating system (i.e., $\mathrm{No}=0$; Sometimes $=2$; Yes=4). This questionnaire has three sub-scales (i.e. physical, emotional, and functional). The scores can range from 0 to 100 and higher scores indicate more disability (e.g. $16-34$ points = mild; 36- 52 points = moderate; and 54+ points = severe) .

- Vertigo symptom scale: (VSS) focuses on symptoms associated with vertigo and to measure the relationship between vertigo, anxiety, and emotional distress in clinical population (Yardley., et al. 1992). VSS is a 28-item self-report questionnaire that is broken down into 3 subscales: functional, ambulatory, and instrumental. The functional subscale assesses a person's perception of basic self-care tasks, the ambulatory subscale assesses mobility-related skills, and the instrumental subscale assesses self-perception in higher-level, more socially sophisticated tasks. The questionnaire requires individuals to rate their self-perceived disability level on a scale that ranges from 1 (independent) to 10 (too difficult, no longer performed).
- Vertigo handicap questionnaire (VHQ): Yardley and Putnam (1999) developed the Vertigo Handicap Questionnaire (VHQ). This questionnaire investigates factors inducing handicap associated to dizziness. The VHQ includes 22 items reproducing the incapacitating results of vertigo, adding restrictions of vertigo on physical and routine activities to impacts on social life and time-out. However, the main limitation of this measure is that the psychometric properties need to be confirmed.

Psychological evaluation:

- $\quad$ To assess the degree to which psychological factors operate in Meniere's disease, two objective personality instruments were administered in a supervised test environment:

- Both the Mental Status Examination and the Sinha's Comprehensive Anxiety Test (SCAT) show a clear picture of the patient having active vestibular symptoms along with High Anxiety Level in his day-to-day life.

- $\quad$ Changes in vertigo over time was maintained by Symptom diary with the help of Excel Spreadsheet.

A datasheet (Appendix A) was compiled to organize the: Case history, Audiological reports, psychological test results and Symptom Diary as documented in the Dept. of Audio-vestibular Sciences and Dept. of Psychology Records.

\section{Clinical findings and interpretation}

1. In PT audiometry results: PTA: Left Ear- 15dBHL; Right Ear68.75dBHL, Provisional Diagnosis: Left Ear: Hearing sensitivity within normal limits and Right Ear: Moderately-severe Sensorineural hearing loss.

2. Speech Audiometry: SRT scores were Left Ear- 20dBHL and Right Ear- 75dBHL. SDS- Left Ear: 90\% and Right Ear: 30\%. SD was low as the hearing thresholds deteriorate, there is progressive decline in the patient's ability to discriminate words.

3. Glycerol test: Patient was given glycerol of $114 \mathrm{ml}$. with equal amount of water $(1.5 \mathrm{ml} / \mathrm{Kg})$. Audiogram and Speech discrimination scores were recorded before and $1.5 \mathrm{hrs}$. Post Glycerol Ingestion. The test result was Positive. Improvement in threshold minimum of $15 \mathrm{~dB}$ in three consecutive octave frequencies with more than 10\% improvement in SDS score in right ear (SDS-50\%) was recorded. 
4. Immittance audiometry: B/L A type tympanogram with acoustic reflex absent in Right ear.

5. SISI test: Rt ear: Score 85\% Positive SISI (suggestive of Cochlear Pathology), Lt ear: Score 10\% Suggestive of intact state of Cochlea and associated parts.

6. OAE: OAEs are an objective, non-invasive and quantitative measure of sensory cell function in the cochlea. It provides information regarding outer hair cell activity, and is regarded as a reliable test for determining the structural basis of a hearing problem, particularly if it involves the outer hair cell system. Results of DP OAE: Right ear: Refer, Left ear: Pass; TOAE: Right ear: Refer, Left ear: Pass. In DPOAEs test, "DP-gram" in which DPOAE amplitudes for a single primary intensity are plotted as a function of frequency of the primary tones were recorded. DPAOEs are sensitive to cochlear pathology such as MD and our result has indicated that DPOAE amplitudes are reduced in the presence of elevated behavioral thresholds indicating affected OHC. Changes in the activity of OHC following glycerol ingestion can also be verified with DPOAE measurements. Concerning monitoring of cochlear function in the glycerol test, it was observed that DPOAE amplitudes increased with hearing improvement following glycerol ingestion supporting the diagnosis.

7. During VNG, A test for vestibular function was carried out as MD was suspected, Video-oculographic recording of eye movements showed normal VOR in vHIT.

8. EcochG: SP/AP ratio was found to be 0.46 (Click stimulus was used in alternative polarity), suggestive of confirmed MD.

9. Quality of life instruments: The QoL questionnaires were completed at baseline, 2 months, 4 months, 6 months, 12 months and 18 months. In Symptom Diary, Vertigo symptoms were rated daily on the symptom diary using a Likert scale from 0 , "no vertigo," to 10, "worst vertigo attack ever". Vertigo rated 2 or greater represented a "counting" vertigo attack, an attack lasting more than 20 minutes. A categorical score was assigned to the patient depending on the level of vertigo improvement during the 2 months of follow-up regardless of treatment assignment. The vertigo control categories served as a representation of severity of patient during the 18-month study.

10. Vertigo handicap questionnaire (VHQ): A score of $75 \%$ was obtained during baseline assessment.
11. The Sinha's comprehensive anxiety test (SCAT) Obtained score was 28 suggestive of moderate to severe anxiety.

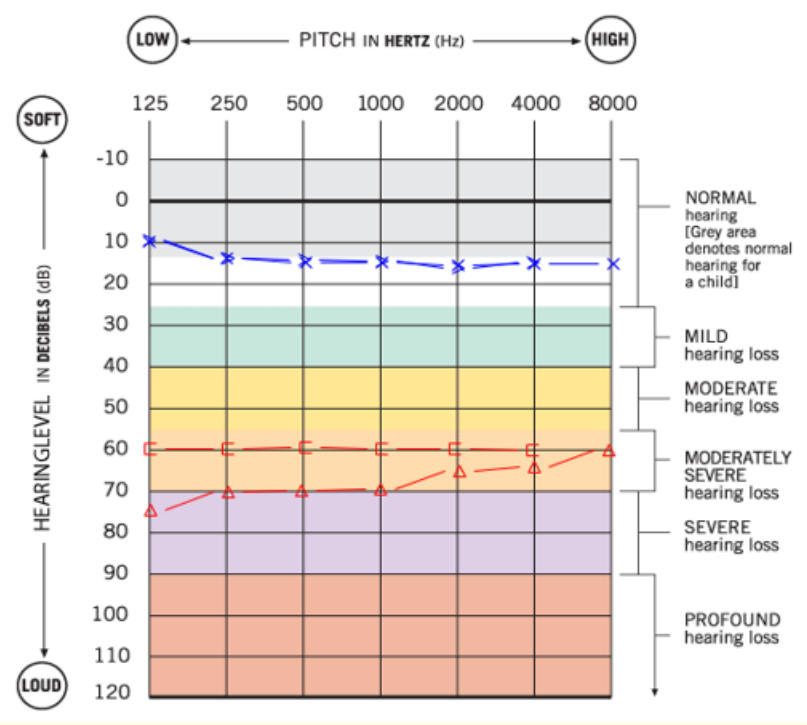

Figure 1: Audiogram of the patient with MD before glycerol ingestion.

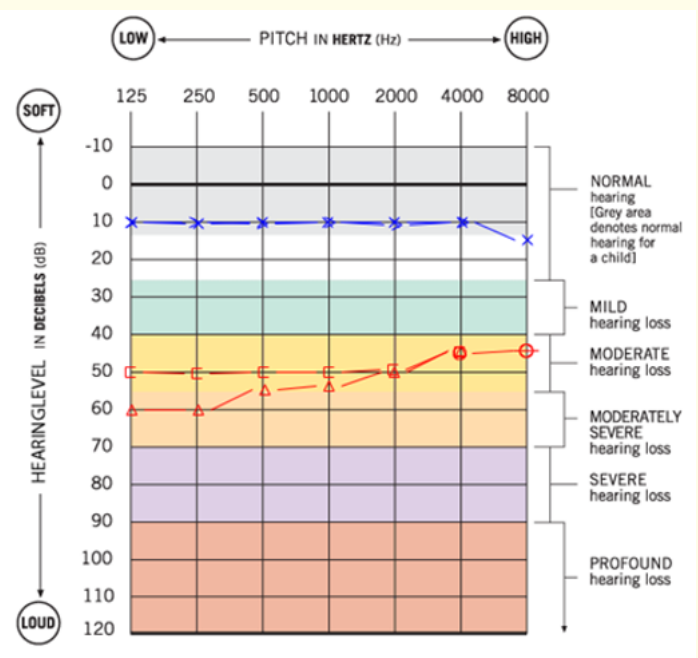

Figure 2: Audiogram of the patient with MD 1.5 hrs. Post glycerol ingestion. 


\begin{tabular}{|l|c|c|c|c|c|c|c|c|c|}
\hline \multicolumn{2}{|l|}{ PTA Thresholds } & $\mathbf{1 2 5 H z}$ & $\mathbf{2 5 0 H z}$ & $\mathbf{5 0 0} \mathbf{~ H z}$ & $\mathbf{1 K H z}$ & $\mathbf{2} \mathbf{K H z}$ & $\mathbf{4 K H z}$ & $\mathbf{8 K H z}$ & $\mathbf{S D S}$ \\
\hline \multirow{2}{*}{ Baseline } & Lt & $10 \mathrm{dBHL}$ & $15 \mathrm{dBHL}$ & $15 \mathrm{dBHL}$ & $15 \mathrm{dBHL}$ & $15 \mathrm{dBHL}$ & $15 \mathrm{dBHL}$ & $15 \mathrm{dBHL}$ & $90 \%$ \\
\cline { 2 - 10 } & Rt & $75 \mathrm{dBHL}$ & $70 \mathrm{dBHL}$ & $70 \mathrm{dBHL}$ & $70 \mathrm{dBHL}$ & $65 \mathrm{dBHL}$ & $65 \mathrm{dBHL}$ & $60 \mathrm{dBHL}$ & $30 \%$ \\
\hline $\begin{array}{l}1.5 \mathrm{hrs} . \text { Post glycerol } \\
\text { ingestion }\end{array}$ & Lt & $10 \mathrm{dBHL}$ & $10 \mathrm{dBHL}$ & $10 \mathrm{dBHL}$ & $10 \mathrm{dBHL}$ & $10 \mathrm{dBHL}$ & $10 \mathrm{dBHL}$ & $15 \mathrm{dBHL}$ & $95 \%$ \\
\cline { 2 - 9 } & Rt & $60 \mathrm{dBHL}$ & $60 \mathrm{dBHL}$ & $55 \mathrm{dBHL}$ & $55 \mathrm{dBHL}$ & $50 \mathrm{dBHL}$ & $45 \mathrm{dBHL}$ & $45 \mathrm{dBHL}$ & $50 \%$ \\
\hline
\end{tabular}

Table 1: Glycerol test.

\section{Multimodal intervention of MD}

1. Medication as a symptomatic treatment: Medication was used to relieve symptoms such as nausea, vomiting and vertigo, but also to prevent further attacks of MD. The anti-vertiginous drugs were prescribed by the ENT.

2. Dietary modification: Low Salt Diet and Diuretics were recommended to the patient. According to Schessel., et al. 1998, Diuretics, anti-vertiginous, antiemetics, sedatives, antidepressants, or herbal medicines may be used to treat allergies, reduce fluid retention, or enhance blood circulation in the inner ear, alleviating the patient's symptoms.

3. Dietary changes: The patient was recommended to eliminate caffeine, alcohol, refined sugars and salt which may help to reduce the frequency and intensity of symptoms. Dietary changes and modifications have proven successful in controlling vertigo.

4. Behavioural therapy: Therapy helping to reduce stress may lessen the severity of the disease symptoms. Counselling forms an important part of treatment in the patient with MD. Medical professionals involved in the treatment of these patients (general practitioners, neurologists, ENTs, psychologists and audiologists) should attempt to break the vicious circle of stress. Such a process started by allocating the necessary time to listen to the patient's complaints, examining the patient's hearing and balance function thoroughly, and explaining to the patient the exact nature of MD. The natural history of the disease was discussed with the patient and their family.

5. Jacobson's progressive muscle relaxation: Progressive muscle relaxation (PMR) is a deep relaxation technique that has been shown to be beneficial in reducing stress and anxiety, as well as relieving insomnia. It is based on the simple principle of tensing (tightening) one muscle group at a time, then relaxing and releasing the tension. PMR is also an excellent tool to help learn about the body and the signals it may be telling you. With practice and time, patient can learn to accu- rately identify and diminish the signs and signals of stress and tension in your body. This technique was recommended to the patient once a day.

6. Hearing rehabilitation: Hearing rehabilitation with the use of hearing aids forms an important part of the treatment of a patient with MD. Widex Unique 330 Fusion, which is a receiver in the ear canal style of hearing aid was prescribed with acclimatization function active (Acclimatization function initially provides a lower level of gain in comparison to actual target gain required as per the initial programming, then automatically the gain is increased to next level after a certain period of time, which is set during programming, so that the patient can get acclimatized to the extra gain gradually, which is very helpful in fluctuating hearing loss. Finally, the target gain is achieved and given automatically after a fixed period of time eliminating the need for reprogramming the hearing aid again and again), Zen program was active so as to continue the tinnitus retraining therapy. The role of the audiologist becomes that of assessing the handicapping effects of hearing impairment in terms of communicative efficiency and evaluating the success of aural rehabilitative procedures in reducing these handicapping effects. The purpose of rehabilitative intervention is to maximize the reception of speech, reduce the impact of psychosocial factors and imparting information about hearing loss, use of hearing aids and communications strategies. When it comes to fitting hearing aids for people who have been diagnosed with MD, there are some main challenges that professionals face. The crucial challenge is the presence of a fluctuating hearing loss (Barber, 1983). Therefore, Follow-up audiograms were recorded. As the hearing loss stabilized in course of 4 months, a hearing aid was be fitted successfully. Due to decreased dynamic area for comfortable speech and SD ability was be affected with the narrowed dynamic range of sound tolerance, proper testing was done along with counselling these problems. The use of hearing aids also showed the advantage of reducing the inconvenience of tinnitus. 
7. Yogic rehabilitation for Meniere's disease: Since yoga helps in increasing the body's overall balance it is also a recommended alternative treatment for ear problems like Meniere's disease. Yoga not only stimulates the neural system, but it also strengthens the labyrinth, commonly known as the inner ear, which is in charge of controlling the body's sense of balance. Certain yoga asanas aid in the smooth and steady movement of fluid through the inner ear canals, which helps to activate the ear's sensors. Malasana (Garland posture) and Ardha Matsyendrasana (Half spinal twist) are two of the most recommended yoga asanas for Meniere's disease. These asanas are highly efficient at restoring inner ear balance. The patient was recommended to stay in this position for at least 30 - 60 seconds.

\section{Results}

The emotional climate was found to have been less cooperative in the family of the patient. Relations between parents were bad more often. Psychic stresses were found also to intensify the symptoms (tinnitus, vertigo and nausea). On the basis of the results, it can be stated that psychological stress resulted from the disease symptoms and may play a role in their precipitation.

While depression is rarely fully addressed in the initial evaluation of otologic symptoms, our results support further investigation and management of the individuals with MD. Psychological assessment, consultation, and the use of antidepressant medications was proved beneficial to the overall wellbeing of the patient and provide an adjunctive treatment for Meniere's disease.

Some noteworthy observation during initial stages were as follows: Re-experiencing Distress/Impairment in social, occupational or other areas of functioning, Intense fear, helplessness, horror Arousal causing sleeping problems, Arousal causing irritability, concentration difficulties, hypervigilance, Avoidance behavior, reduced interest/participation in activities, feeling detached from others, Sense of foreshortened future etc.

Our results showed that pharmacological treatment, Dietary change and modification helped in symptomatic intervention, $\mathrm{Au}$ dio-vestibular Rehabilitation played a major role in taking care of Hearing Loss and Tinnitus with the help of accurate prescription of hearing aids with fine tuning according to the need of the patient significantly improving vertigo and hearing ability in patients, Yogic Rehabilitation with psychological counselling played a major role in patient's life in maintaining positive attitude in life along with healthy habits and schedule.

\section{Discussion}

Physicians treating patients with Meniere's disease are struck by the magnitude of the psychological effect that the disease has on those who are afflicted [3]. Only Objective measures including Videonystagmography, Audiometry, or electrocochleography may have been used to assess the effectiveness of a particular intervention for Meniere's disease.

The extreme variability in the symptoms of Meniere's disease coupled with patient variables such as coping skills, family support and vocational requirements may cause a tolerable amount of vertigo in one patient to become disabling in another patient. This effect is best measured through QoL measures.

The functionality levels assessed by the general questionnaires on QOL are helpful but do not go far enough at assessing the life impact of the disease. The functionality levels require generalization with regard to activity levels, necessity of changing planned activities, and disability status over a period of time [5]. A single vertigo spell may be disabling for an airline pilot but may have less life impact for a pilot who has already retired. Even the most steadfast patient experiences emotional tiredness and intolerance as the episodes become more frequent.

The gold standard for assessing the success or failure of a treatment for Meniere's disease is the frequency and severity of vertigo as recorded on a daily symptom report card. The frequency and severity of vertigo spells are associated to tinnitus, HL, auditory fullness, and QoL. Evaluating the basis for the interrelationship between a patient's vestibular and psychiatric symptoms and providing the patient with an adequate explanation for their MD is a cornerstone in managing such patients. Furthermore, prompt vestibular rehabilitation therapy appears to be critical in managing individuals who report with both dizziness and anxiety [6]. Better management of the psychological problems of people with MD has the potential to improve quality of life when physical symptoms cannot be controlled, and to prevent the development of psychological disorders [7]. Reducing psychological distress may also help to relieve physical symptoms, since there is evidence that anxiety and avoidance behavior may augment and prolong vestibular-related symptoms.

\section{Conclusion}

Physicians should consider multimodal treatment strategies for Meniere's disease patients. In light of the results from this case 
study, there are two points that we should keep in mind when we treat patients with Meniere's disease. First, we should assess the patient with Meniere's Disease in a Multi-disciplinary approach having teamwork and regular meetings to discuss progress.

Secondly, we should consider various treatment strategies ranging from drug administration to Yogic Rehabilitation, before Meniere's patients with a long duration of disease develop mental illness.

\section{Acknowledgements}

This study was supported by the Institute of Health Sciences, Bhubaneswar, India and is part of the research programme of our Department of Audiology and Speech-Language, Department of Psychology, and Department of Yogic Rehabilitation.

\section{Conflict of Interest}

The authors report no conflicts of interest. The authors alone are responsible for the content and writing of the paper.

\section{Bibliography}

1. Coker Newton J., et al. "Psychological profile of patients with Meniere's disease". Archives of Otolaryngology-Head and Neck Surgery 115.11 (1989): 1355-1357.

2. Furukawa Masashi., et al. "Psychological condition in patients with intractable Meniere's disease". Acta Oto-Laryngologica 133.6 (2013): 584-589.

3. Green Jr, et al. "Quality of life instruments in Ménière's disease". The Laryngoscope 117.9 (2007): 1622-1628.

4. van Cruijsen Nynke., et al. "Analysis of cortisol and other stress-related hormones in patients with Meniere's disease". Otology and Neurotology 26.6 (2005): 1214-1219.

5. Fowler Edmund P and Adolf Zeckel. "Psychosomatic aspects of Meniere's disease". Journal of the American Medical Association 148.15 (1952): 1265-1268.

6. Jacob Rolf G and Joseph M Furman. "Psychiatric consequences of vestibular dysfunction”. Current Opinion in Neurology 14.1 (2001): 41-46.
7. Kirby Sarah E and Lucy Yardley. "Understanding psychological distress in Meniere's disease: a systematic review". Psychology, Health and Medicine 13.3 (2008): 257-273.

Volume 3 Issue 10 October 2021

(C) All rights are reserved by Satyabrata Panigrahi., et al. 